\title{
Thrombotic thrombocytopenic purpura: a rare case presenting with splenic infarction
}

\begin{abstract}
Microangiopathic hemolytic anemia (MAHA), thrombocytopenia, fever, renal failure, and neurologic symptoms comprise the cardinal features of thrombotic thrombocytopenic purpura (TTP). Etiologies can include medications, infections, cancers, or transplantation. However, recognition of thrombotic thrombocytopenic purpura can be difficult because of the variety of presentations and lack of specific diagnostic criteria. We are presenting a case of acute TTP following a bout of splenic infarction. This rare case reminds us that splenic infarction can be an atypical presentation of TTP. Prompt recognition of TTP is important because the disease responds well to plasma-exchange treatment which improves patient's prognosis, and is associated with a high mortality rate when untreated.
\end{abstract}

Keywords: thrombotic thrombocytopenic purpura, splenic infarction, microangiopathic hemolytic anemia (MAHA)
Volume 2 Issue 3 - 2016

\author{
Murad Baba,' Luis W Dominguez, ${ }^{2}$ Amit \\ Patel $^{3}$ \\ 'Department of Internal Medicine, Jersey City Medical Center, \\ USA \\ ${ }^{2}$ Department of Hematology/Oncology, Jersey City Medical \\ Center, USA
}

Correspondence: Murad Baba, Department of Internal Medicine, Jersey City Medical Center, 355 Grand Street, Jersey City-NJ 07302, USA, Fax +2019152219,

Email muurad@hotmail.com

Received: February 07, 2016 | Published: May 16, 2016
Abbreviations: TTP, thrombotic thrombocytopenic purpura; HUS, hemolytic uremic syndrome; ITP, idiopathic thrombocytopenic purpura; DIC, disseminated intravascular coagulation; FFP, fresh frozen plasma; $v W F$, vonwillibrand's factor; MRI, magnetic resonance imaging

\section{Introduction}

Thrombotic Thrombocytopenic Purpura (TTP) is a hypercoagulable state, in which platelets aggregate and clot in the microvasculature. The majority of cases can be linked to ADAMST13 deficiencies, a protein responsible for cleaving VonWillibrand's Factor (vWF). Hypo functional ADAMST13 results in un-mitigated vWF activity, leading to excessive platelet aggregation. Increased clotting leads to decreased circulating platelets, hemolysis and anemia. Microvascular infarctions cause non-blanching purpura, renal damage, and cerebral ischemia resulting in a myriad of Central Nervous System manifestations from altered mental status to seizures. While some cases are idiopathic, $10 \%$ of TTP diagnoses are associated with sepsis ${ }^{1}$ or malignancy ${ }^{2}$ as well as enterocolitis, especially from $E$. coli. ${ }^{3}$ Originally, TTP was characterized by a classic pentad of microangiopathic anemia, thrombocytopenia, fever, neural manifestations, and renal damage. ${ }^{4}$ However, too many cases were missed so a more liberal diagnostic triad was created: microangiopathic anemia associated fragmented erythrocytes, and thrombocytopenia. ${ }^{5}$

\section{Case description}

79year old Hispanic male with past medical history of HTN and osteoarthritis presented to the emergency room complaining of left side abdominal pain for three days and intermittent confusion. Physical examination was unremarkable apart from fluctuating mental status and left lower quadrant tenderness on palpation of abdomen. Initial laboratory investigations revealed anemia, thrombocytopenia, elevated bilirubin but normal liver enzymes and lipase. The peripheral blood smear showed schistocytes. Imaging studies including abdominal Computed Tomography (CT) scan demonstrated a wedge- shaped hypo-enhancing lesions in the spleen typical for splenic infarcts as shown in Figure 1, and a brain CT scan was unremarkable.

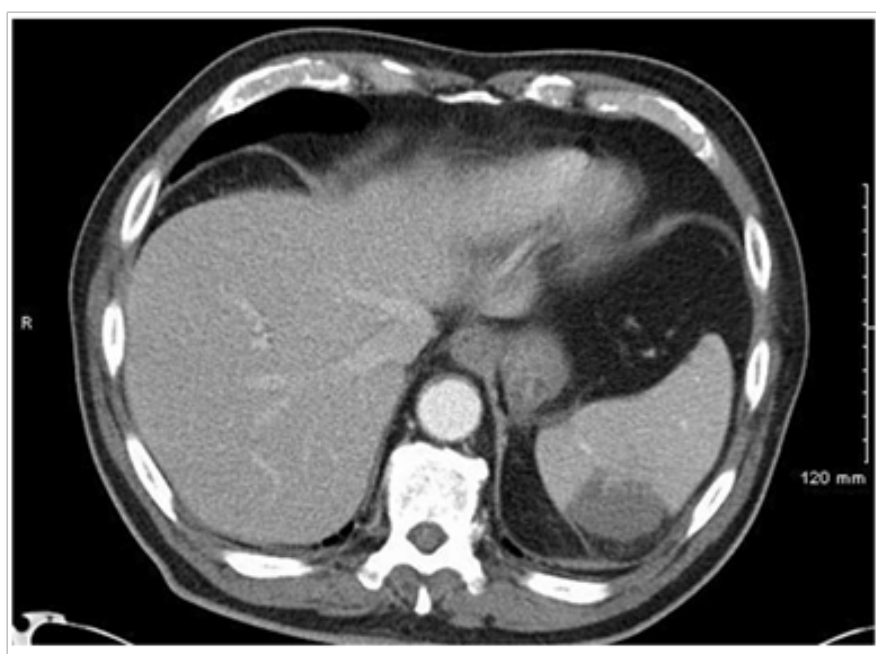

Figure I Computed Tomography scan of the Abdomen showing wedgeshaped hypo-enhancing lesion in the spleen.

Plasma exchange was initiated immediately along with systemic steroids. Platelets counts improved, the patient remains afebrile and hemodynamically stable but his altered mental status persisted. Further workup for his persistent change in mental status revealed multiple bilateral acute lacunar infarcts on Magnetic Resonance Imaging (MRI) of the Brain as shown in Figure 2, which is likely embolic in etiology. After six days, the patient became lethargic, febrile, tachycardia and the platelets count dropped again. Plasma exchange subsequently stopped when positive blood cultures grew a gram-negative bacteria $(E$. coli). The patient progressively improved following therapy with cefepime and gentamycin. Plasma exchange resumed and he maintained acceptable platelet count until being discharged from the hospital. 


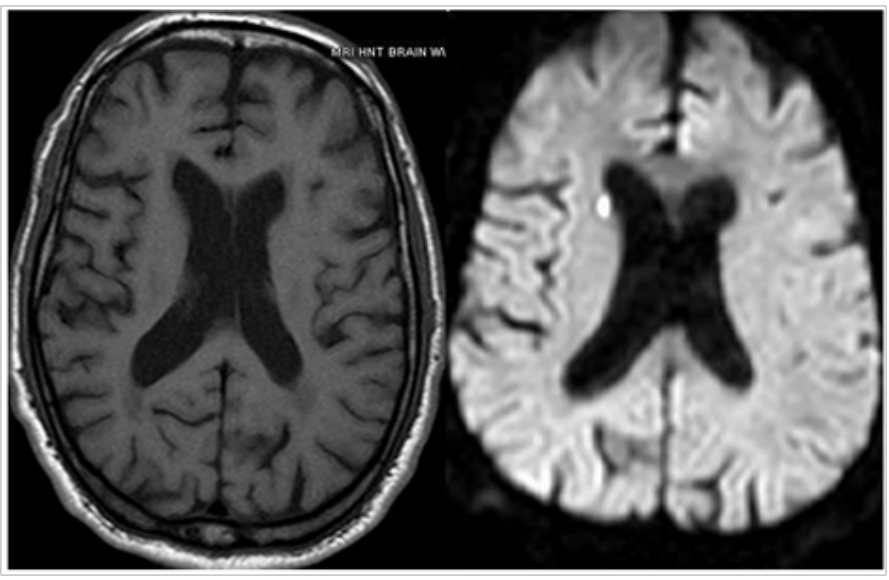

Figure 2 Axial DWI Magnetic Resonance Imaging of the Brain with and without contrast showing acute lacunar infarct.

\section{Discussion}

\section{Epidemiology}

TTP is estimated to have prevalence between 4-11cases per million. ${ }^{6}$ When untreated, TTP has a mortality rate of $90 \%{ }^{7}$ However, treated TTP has a survival rate of up to $80 \%{ }^{7}$ As TTP is a thrombotic disease, the most common and feared causes of death are cerebral, renal and myocardial infarction. ${ }^{4}$ Fifty $\%$ of cases will have central nervous complications, such as seizures, ${ }^{8}$ and some cases have even reported neural sequelae preceding hematological findings ${ }^{9}$. Renal injury (indicated by elevated creatinine) occurs in $33 \%$ of patients. While TTP is thought of as an acute process, $25 \%$ of cases can be indolent, progressing over weeks. ${ }^{7}$ With regards to laboratory findings, $95 \%$ of TTP cases will have thrombocytopenia with platelet counts $<60,000 .{ }^{9}$ Evidence of hemolysis should be found in the form of hyperbilirubinemia, elevated LDH, schistocytes on peripheral smear, and anemia.

\section{Diagnostic work-up}

The differential diagnosis for TTP includes Hemolytic Uremic Syndrome (HUS), Idiopathic Thrombocytopenic Purpura (ITP), Disseminated Intravascular Coagulation (DIC), HELLP syndrome in pregnant patients, as well as secondary causes of TTP. Some drugs are known to cause TTP, such as mitomycin, gemcitabine, and of course anti-platelet drugs such as clopidogrel and ticlopidine. ${ }^{10}$ HUS more commonly has dramatic renal involvement, is linked to GI infections, and is not treated by plasma exchange. ${ }^{11}$ DIC will have prolonged PTT and INR when compared to TTP. ITP will often lack the neurological sequelae indicative of TTP.

\section{Treatment}

The first line treatment for TTP is plasma exchange. The objective is to remove the auto antibodies, and replace them with Fresh Frozen Plasma (FFP). ${ }^{12}$ Complete plasma exchange, replacing $1-1.5 \mathrm{x}$ the patient's calculated volume occurs daily until platelets and organ function normalize. ${ }^{13}$ This can often take weeks, as the neurological sequelae resolve, then the anemia, and finally renal normalization. $\mathrm{LDH}$ is also a keen marker for treatment response, as it indicates hemolysis and tissue damage. ${ }^{14}$ If plasma exchange is not possible, FFP infusions are an acceptable bridging therapy until the patient can be transferred..$^{15}$
Twenty percent of patients will not respond to plasma exchange, ${ }^{16}$ making our first line treatment significantly flawed. ${ }^{17}$ On a side note, it is postulated that the majority of patients who do not respond to plasma exchange, developed TTP from atypical etiologies such as malignancy. ${ }^{17}$ As a second line treatment and for severe cases, immuno suppressants are used. The mainstays of this treatment are steroids and rituximab. ${ }^{17}$ Response to treatment with rituximab is usually seen within fourteen days. ${ }^{18}$ After platelets have normalized, treatment should be continued for at least 2 more days to insure efficacy. ${ }^{6,13}$ Given the intensity of the treatment, it is not surprising that $26 \%$ of patients develop major complications, including infections, venous clots, and hypotension. Two percent develop life-threatening conditions such a catheter-site hemorrhage, and catheter-related sepsis. ${ }^{19}$

TTP is a rare but potentially fatal disorder, prompt recognition is important because the disease responds well to plasma-exchange treatment, but is associated with a high mortality rate when untreated. In the era before effective treatment with plasma exchange, 90 percent of patients with thrombotic thrombocytopenic purpura died from systemic microvascular thrombosis that caused cerebral and myocardial infarctions and renal failure. However, recognition of thrombotic thrombocytopenic purpura can be difficult because of the variety of presentations and lack of specific diagnostic criteria. ${ }^{7}$

\section{Conclusion}

Our patient presented with abdominal pain secondary to a splenic infarct. Eighty-eight percent of splenic infarcts are caused by hematological problems such as blood cancers, hypercoagulable states, atrial fibrillation and vasculitides..$^{20}$ However, very few have reported TTP as a precipitant. Indeed, this case is remarkable as a rare disease with an even more rare presentation. Given the severity, the patient was started immediately on steroids and plasma exchange. The brain MRI revealed one of the dreaded complications listed above: multiple, bilateral lacunar infarcts. Finally, our patient also developed a feared, predictable life threatening complication to the therapy-nosocomial E. Coli sepsis. Despite the myriad of set-backs, our patient improved and was discharged home.

This case stands apart as a rare presentation of a rare disease. Furthermore, it is a classic example of the importance of vigilance in treating a complex condition, with complications from both the disease, and the treatment. Early identification of TTP and anticipation of the adverse effects of treatment are vital in avoiding the significant morbidity and mortality of this potentially debilitating disease.

\section{Acknowledgements}

None.

\section{Conflict of interest}

The authors have no conflicts of interest to declare relevant to this article.

\section{References}

1. Booth KK, Terrell DR, Vesely SK, et al. Systemic infections mimicking thrombotic thrombocytopenic purpura. Am J Hematol Sep. 2011;86(9):743-751.

2. George JN, Vesely SK, Terrell DR. The oklahoma thrombotic thrombocytopenic purpura-hemolytic uremic syndrome (TTP-HUS) Registry: a community perspective of patients with clinically diagnosed TTP-HUS. Semin Hematol. 2004;41(1):s60-s67. 
3. Fischer LJ, Weyant RS, White EH, et al. Intracellular multiplication and toxic destruction of cultured macrophages by Capnocytophaga canimorsus. Infect Immun. 1995;63(9):3484-3490.

4. Amorosi EL, Ultmann JE. Thrombotic thrombocytopenic purpura: report of 16cases and review of the literature. Medicine. 1966;45(2):139-159.

5. Brain MC, Dacie JV, Hourihane Do. Microangiopathic haemolytic anemia: the possible role of vascular lesions in pathogenesis. $\mathrm{Br} J$ Haematol. 19622;8:358-374.

6. Terrell DR, Williams LA, Vesely SK, et al. The incidence of thrombotic thrombocytopenic purpura-hemolytic uremic syndrome: all patients, idiopathic patients, and patients with severe ADAMTS-13 deficiency. $J$ Thromb Haemost. 2005;3(7):1432-1436.

7. George JN. Clinical practice. Thrombotic thrombocytopenic purpura. $N$ Engl J Med. 2006;354(18):1927-1935.

8. Vesely S, George JN, Lämmle B, et al. ADAMTS13 activity in thrombotic thrombocytopenic purpura-hemolytic uremic syndrome: relation to presenting features and clinical outcomes in a prospective cohort of 142 patients. Blood. 2003;102(1):60-68.

9. Rojas JC, Banerjee C, Siddiqui F, et al. Pearls and oy-sters: acute ischemic stroke caused by atypical thrombotic thrombocytopenic purpura. Neurology. 2013;80(22):235-238.

10. Medina PJ, Sipols JM, George JN. Drug-associated thrombotic thrombocytopenic purpura-hemolytic uremic syndrome. Curr Opin Hematol. 2001;8(5):286-293.

11. Moake JL. Thrombotic microangiopathies. $N$ Engl $J$ Med. 2002;347(8):589-600.

12. Raife TJ, Friedman KD, Dwyre DM. The pathogenicity of von Willebrand factor in thrombotic thrombocytopenic purpura: reconsideration of treatment with cryopoor plasma. Transfusion. 2006;46(1):74-79.
13. Allford SL, Hunt BJ, Rose P, et al. Guidelines on the diagnosis and management of the thrombotic microangiopathic haemolytic anemias. Br J Haematol. 2003;120(4):556-573.

14. Cohen JA, Brecher ME, Bandarenko N. Cellular source of serum lactate dehydrogenase elevation in patients with thrombotic thrombocytopenic purpura. J Clin Apher. 1998;13(1):16-19.

15. Duffy SM, Coyle TE. Platelet transfusions and bleeding complications associated with plasma exchange catheter placement in patients with presumed thrombotic thrombocytopenic purpura. J Clin Apher. 2013;28(5):356-358.

16. Haas M, Leko-Mohr Z, Lang T, et al. The LDH ratio as a marker for response to plasma exchange in HUS/TTP of the adult. Clin Nephrol. 2002;57(6):414-420.

17. Li GW, Rambally S, Kamboj J, et al. Treatment of refractory thrombotic thrombocytopenic purpura with $\mathrm{N}$-acetylcysteine: a case report. Transfusion. 2014;54(5):1221-1224.

18. Tun NM, Villani GM. Efficacy of rituximab in acute refractory or chronic relapsing non-familial idiopathic thrombotic thrombocytopenic purpura: a systematic review with pooled data analysis. J Thromb Thrombolysis. 2012;34(3):347-359.

19. Howard MA, Williams LA, Terrell DR, et al. Complications of plasma exchange in patients treated for clinically suspected thrombotic thrombocytopenic purpura-hemolytic uremic syndrome. Transfusion. 2006;46(1):154-156.

20. Nores M, Phillips EH, Morgenstern L, et al. The clinical spectrum of splenic infarction. Am Surg. 1998;64(2):182-188. 